\title{
Binomial and Poisson Confidence Intervals and its Variants: A Bibliography
}

\author{
Anwer Khurshid \\ Department of Mathematical and Physical Sciences \\ College of Arts and Science \\ University of Nizwa \\ P. O. Box 33, PC 616 \\ Birkat Al Mouz, Oman \\ anwer_khurshid@yahoo.com \\ Mohammed I. Ageel \\ Department of Mathematics \\ Faculty of Science \\ King Khalid University \\ Abha, Saudi Arabia \\ miaqeel@kku.edu.sa
}

\begin{abstract}
The binomial and Poisson distributions are basis to many aspects of statistical data analysis. This bibliography attempts to provide a comprehensive listing of available literature on calculating confidence intervals for binomial and Poisson distributions and its variants. The bibliography includes articles published in statistical and subject-matter journals and in conference proceedings.
\end{abstract}

Keywords: binomial confidence interval; Poisson confidence interval; Bibliography.

\section{Introduction}

In statistical applications, we generally aren't satisfied with just quoting a 'best estimate' for the value of a parameter. We would also like to know how precise the estimate is, or, alternatively, give some feeling for how close the point estimate may be expected to be to the true value of the unknown parameter. This brings us to the topic of interval estimation.

One task in the analysis of data is to construct a confidence interval for the parameter(s) for the distribution. A confidence interval is a range around a measurement that conveys how accurate the measurement is. Statisticians use a confidence interval to explain the amount of uncertainty associated with a sample estimate of a population parameter. The concept of confidence intervals was advanced independently by Fisher (1935) and Neyman (1937) [see also Clopper and Pearson, 1934; Neyman 1935; 1941 for some discussion]. Fisher coined the term 'fiducial limits' and Neyman used the name 'confidence intervals'. At present confidence intervals are more frequently used in the scientific literature.

Two of the most widely used discrete probability distributions are the binomial and Poisson and it crops up so frequently in such diverse applications that we 
need no further justification for emphasizing it. Undoubtedly, the statistical procedures based on binomial distribution remain one of the easiest and most useful kinds of procedures in the statistical repertoire of the researcher. The Poisson distribution has also found an extensive and varied application in formulating probability models for a wide variety of phenomena in the sciences. In addition to being important in its own regard, the binomial and Poisson distributions are the sources of many statistical properties calculated from mortality and disease data. In many epidemiologic studies the Poisson distribution is usually associated with rare diseases and plays an important role in analyzing discrete data. In epidemiology the inferences on the standard mortality ratio (SMR) are based on the Poisson probability model.

To date, there had been many discussions by various authors on the construction of both point and interval estimates for binomial and Poisson distributions. Many different methods had been proposed for estimation of confidence intervals for these distributions, some working well in general situations, others only in special cases. It is however common that the effectiveness of these intervals mainly hinges on some properties of its distribution, which render it less attractive. Johnson et al. (2005) provide a wealth of information on binomial and Poisson distribution and their applications. There are specialized books listed in the References Cited, where in authors' view, a substantive discussion of confidence intervals for binomial and Poisson distributions and their variants have been made.

The literature on binomial and Poisson confidence intervals and its variants is diverse, appears in a variety of journals from different specialties, and most papers cite only a very limited part of it. Before one embarks on a research project in this area, it is imperative to know what has been done previously. So far there does not exist any publication to serve this purpose and the present bibliography is intended to fill this void. It is the intention of this bibliography to provide a complete list of all references dealing with methodological as well applied aspects of the binomial and Poisson confidence intervals.

\section{Methods, Scope and Coverage}

To locate relevant material for inclusion in the bibliography, we used a variety of publications, including annual series that index published material in the field (Current Index to Statistics, Statistical Theory and Methods Abstracts) proceedings of conferences and workshops, and journals.

The following guidelines and criteria for inclusion were followed in the compilation of this work.

(i) The bibliography confines itself to only those works specifically concerned with the confidence intervals for binomial and Poisson distributions and their variants. Publications on confidence intervals in general are not covered. Some restricted material on confidence intervals that may have implications is included in a haphazard or unsystematic way. 
(ii) Confidence intervals for binomial and Poisson distributions are interpreted in a broad sense. Thus, the bibliography includes not only works about confidence intervals for binomial and Poisson distributions in general, but also articles dealing with expository material, technical notes, and examples appropriate for use either as direct or indirect resource material or to supplement and enrich the presentation of standard textbooks.

(iii) Books are generally not included in this bibliography, however, pertinent articles extracted from anthologies, edited books, yearbooks, conference proceedings, and so forth are included.

(iv) The coverage of journals varies from one journal to another. Among statistical journals the coverage is fairly complete for those journals which are regularly scanned in their entirety by the Current Index to Statistics. Relevant items from this publication were located using keywords such as binomial, Poisson, confidence intervals etc.

(v) The part of bibliography contains references from non-statistical journals, such as those in social, physical, biological, medical, epidemiological, engineering sciences. Among non-statistical journals, the coverage is highly selective in nature and only those entries dealing with the confidence intervals for binomial and Poisson distributions in general are included. For journals not regularly scanned the coverage is inconsistent and unsystematic and only those items which came to our attention by random browsing of selected issues of a wide variety of journals are included. The nature of this selection was further restricted and biased by our own specific interests in particular substantive fields and availability of journals in our libraries.

(vi) Few papers presented at professional meetings and conferences, but not available in the form of published proceedings, are included.

\section{Description of Entries}

Entries are alphabetized according to the first author's last name. Multipleauthored papers are listed under the first-named author only. For each author, the items of which he/she is the sole author are listed first, ordered by year. These are followed by the dual-authored items, and then the multiple-authored items, of which he/she is the senior author. The language of the articles, if known to be other than English, is indicated after the title of the article.

Preparation of a bibliography, however comprehensive, is never complete, since quite a few articles remain unpublished and some are published in obscure journals not readily accessible. The authors have striven to make the bibliography's coverage as complete as possible. It is certain, however, that other relevant papers have unintentionally been left out. The authors would, therefore, welcome suggestions of further papers pertinent to this subject for inclusion in a possible supplement. 
In order to save space, abbreviations for journals, periodicals, proceedings, and other publications cited in the bibliography, similar to the ones employed by the Current Index to Statistics, have been introduced. A list of the abbreviations and complete names of the publications is given as follows:

\begin{tabular}{|c|c|}
\hline Abbreviations & Complete Names \\
\hline ActAppMath & Acta Applicandae Mathematicae \\
\hline AdvAppErg & $\begin{array}{l}\text { Advances in Applied Ergonomics, A. F. Ozok and G. Salvendry } \\
\text { (Eds.), USA Publishing, West Lafayette, IN. }\end{array}$ \\
\hline AdvAppStat & Advances and Applications in Statistics \\
\hline AmerJEpid & American Journal of Epidemiology \\
\hline AmerStat & The American Statistician \\
\hline AMIAAnSymPro & AMIA Annual Symposium Proceedings \\
\hline AnallnsStMa & Annals of Institute of Statistical Mathematics \\
\hline AnalStat & Annals of Statistics \\
\hline AnlsEmMed & Annals of Emergency Medicine \\
\hline AnlsMathStat & Annals of Mathematical Statistics \\
\hline AppEcoEnvRes & Applied Ecology and Environmental Research \\
\hline AppMathComp & Applied Mathematical Computation \\
\hline ASAProBiop & $\begin{array}{l}\text { ASA Proceedings of Biopharmaceutical Section, American } \\
\text { Statistical Association, Alexandria, VA }\end{array}$ \\
\hline ASAProJSM & ASA Proceedings of the Joint Statistical Meetings \\
\hline ASAProStatEdu & $\begin{array}{l}\text { ASA Proceedings of Statistical Education Section, American } \\
\text { Statistical Association, Alexandria, VA }\end{array}$ \\
\hline ASAProSurResMeth & $\begin{array}{l}\text { ASA Proceedings of Survey Research Methods Section, American } \\
\text { Statistical Association, Alexandria, VA }\end{array}$ \\
\hline AustJStat & Australian Journal of Statistics \\
\hline AustNZJStat & Australian and New Zealand Journal of Statistics \\
\hline Autca & Automatica \\
\hline Biometrika & Biomka \\
\hline BiomtrcJ & Biometrical Journal \\
\hline Biomtrcs & Biometrics \\
\hline Biostats & Biostatistics \\
\hline BMJ & British Medical Journal \\
\hline CalStAssBull & Calcutta Statistical Association Bulletin \\
\hline CanJStat & Canadian Journal of Statistics \\
\hline CMAI & Canadian Medical Association Journal \\
\hline ClinOnc & Clinical Oncology \\
\hline CommStA & Communications in Statistics: Theory and Methods \\
\hline CommStB & Communications in Statistics: Simulation and Computation \\
\hline CompBioMed & Computers in Biology and Medicine \\
\hline CompBioRsr & Computers in Biomedical Research \\
\hline CompStDAna & Computational Statistics and Data Analysis \\
\hline CompStQ & Computational Statistics Quarterly \\
\hline ConCliTri & Controlled Clinical Trials \\
\hline ConlnfTheo & $\begin{array}{l}\text { Conference on Information Theory, Statistical Decision Functions, } \\
\text { Random Processes. B.: Transactions, Prague, D. Reidel } \\
\text { Publishing Co., Dordrecht; Hingham, MA }\end{array}$ \\
\hline DrlnfJ & Drug Information Journal \\
\hline EncyBiost & $\begin{array}{l}\text { Encyclopedia of Biostatistics, Second Edition, P. Armitage and T. } \\
\text { Colton (Eds.), Wiley and Sons, Inc., New York, U.S.A. }\end{array}$ \\
\hline
\end{tabular}


EncyStatBehSci

EncyStatSc

EnvHeaPer
EuJEpi
Epid
FEJTStat
HacBNatEng
IndQCon
IntRevPsi
IntJEpid
IntJStatManSys
JABES
JAES
JAMA
JapJAppStat
JapJBiom
JAppStat
JASA

JASS

JBiopharmStat

JEcoEnt

JEpiCommHealth

JFacEngSeiUni

JJapSocCStat

JMissASci

JMM

JModAppStMeth

JOccuHealth

JOrtho

JouJStaSoc

JQualTek

JRSS-A

JRSS-B

JRSS-C

JStatCompSim

JStatPlalnf

LeoEJPrac

LeoJSci

MathMStat

MathScntst

MemShig

Metron

Midwif

ModAssStApp

NavResLog

NeurEpid

NuclMPhyRsr

PEngRemSen

PhilTran
Encyclopedia of Statistics in Behavioral Science, B. Everitt and D. Howell (Eds.), Wiley and Sons, Inc., New York, U.S.A.

Encyclopedia of Statistical Sciences, S. Kotz and N.L. Johnson (Eds.), John Wiley and Sons, Inc., New York, U.S.A.

Environmental Health Perspectives

European Journal of Epidemiology

Epidemiology

Far East Journal of Theoretical Statistics

Hacettepe Bulletin of Natural Sciences and Engineering

Industrial Quality Control

Interdisciplinaria Revista de Psicologia y Ciencias Afines

Internet Journal of Epidemiology

International Journal of Statistics and Management System

Journal of Agricultural, Biological and Environmental Statistics

Journal of Audio Engineering Society

Journal of the American Medical Association

Japanese Journal of Applied Statistics

Japan Journal of Biometry

Journal of Applied Statistics

Journal of the American Statistical Association

Journal of Applied Statistical Science

Journal of Biopharmaceutical Statistics

Journal of Economic Entomology

Journal of Epidemiology and Community Health

Journal of the Faculty of Engineering, Seiki University, Japan

Journal of the Japanese Society of Computational Statistics

Journal of the Mississippi Academy of Sciences

Journal of Microbiological Methods

Journal of Modern Applied Statistical Method

Journal of Occupational Health

Journal of Orthodontics

Journal of the Japan Statistical Society

Journal of Quality Technology

Journal of the Royal Statistical Society, Series A

Journal of the Royal Statistical Society, Series B

Journal of the Royal Statistical Society, Series C

Journal of Statistical Computation and Simulation

Journal of Statistical Planning and Inference

Leonardo Electronic Journal of Practices and Technologies

Leonardo Journal of Sciences

Mathematical Methods of Statistics

Mathematical Scientist

Memoirs of the Faculty of Education, Shiga University; Natural

Science and Pedagogic Science

Metron

Midwifery

Model Assisted Statistics and Applications

Naval Research Logistics

Neuroepidemiology

Nuclear Instruments and Methods in Physics Research, Series A

Postgraduate Engineering and Remote Sensing

Philosophical Transactions 


\begin{tabular}{|c|c|}
\hline PhyMed & Physica Medica \\
\hline ProcAmerMathSoc & Proceedings of American Mathematical Society \\
\hline ProcFifthBerk & $\begin{array}{l}\text { Proceedings of Fifth Berkeley Symposium on Mathematical } \\
\text { Statistics and Probability, University of California Press, California. }\end{array}$ \\
\hline ProcMIE2000 & $\begin{array}{l}\text { Proceedings of the MIE2000 and GMDS2000, A. Hasman, B. } \\
\text { Blobel, J. Dedeck, R. Engelbrecht, G. Gell and H. -U. Prokosch } \\
\text { (Eds.). IOS Press, Amsterdam. }\end{array}$ \\
\hline ProcSASUsGr & $\begin{array}{l}\text { Proceedings of the SAS Users Group International Conference, } \\
\text { SAS Institute, Inc. Cary, NC. }\end{array}$ \\
\hline ProcSixthStatSemin & $\begin{array}{l}\text { Proceedings of Sixth Statistics Seminar, University of Karachi, } \\
\text { Pakistan }\end{array}$ \\
\hline PubOpRes & $\begin{array}{l}\text { Public Opinion Research Focus, L. O. Petrieff and R. V. Miller } \\
\text { (Eds.). Nova Publishers, Hauppauge, NY. }\end{array}$ \\
\hline QualQuan & Quality and Quantity \\
\hline RevColEst & Revista Colombiana de Estadística \\
\hline RevIntStIns & Review of the International Statistical Institute \\
\hline RevMatEst & Revista de Matemática e Estatistica \\
\hline REVSTAT & REVSTAT Statistical Journal \\
\hline RevStatApp & Revue de Statistique Appliquee \\
\hline RJou & The R Journal \\
\hline RptJUSE & $\begin{array}{l}\text { Reports of Statistical Application and Research, Japanese Union } \\
\text { of Scientists and Engineers }\end{array}$ \\
\hline SciWorJ & Scientific World Journal \\
\hline SoAfrJStat & South African Journal of Statistics \\
\hline SocMeth & $\begin{array}{l}\text { Sociological Methodology, C. C. Clogg (Ed.). American } \\
\text { Sociological Association, Washington, D. C. }\end{array}$ \\
\hline StatComp & Statistics and Computing \\
\hline StatConf & $\begin{array}{l}\text { Statistics with Confidence, Confidence Intervals and Statistical } \\
\text { Guidelines, M. J. Gardner and D. G. Altman (Eds.). BMJ } \\
\text { Publishing Group, London }\end{array}$ \\
\hline StatMed & Statistics in Medicine \\
\hline StatMedRsr & Statistical Methods in Medical Research \\
\hline StatNeer & Statistica Neerlandica \\
\hline StatPap & Statistical Papers \\
\hline StatProbLett & Statistics and Probability Letters \\
\hline Stats & Stats \\
\hline StatSc & Statistical Science \\
\hline StatSin & Statistica Sinica \\
\hline Ststcian & The Statistician \\
\hline TeachgSt & Teaching Statistics \\
\hline TechEng & Techniques d'Enquete \\
\hline Technmcs & Technometrics \\
\hline ThePrApp & Theory of Probability and Its Applications \\
\hline TrAmMaSo & Transactions of American Mathematical Society \\
\hline TranElecComm & Transactions on Electronics and Communications \\
\hline
\end{tabular}

Finally, in attempt to assist the users of the bibliography in their search for specific references of particular topics and interest, the following letter codes are used to classify each entry. 


\section{Code Explanation of Topic}

A. Confidence intervals/limits

A1. General

A2. Rate(s) / ratio(s) / proportion(s) / probabilities

A2.1 Of two rates

A2.2 Of two proportions / two probabilities

A3. Difference(s)

A3.1 Of two proportions / probabilities

A3.2 In correlated binary proportions

A3.3 Between two independent proportions

A3.4 Between matched proportions

A4. Relative risk / odds ratio / risk ratio / relative difference / rate ratio

A5. Simultaneous / sequential

A6. Fiducial intervals / limits

A7. Generalized confidence intervals

A8. Number needed to treat (reciprocal of the difference of two proportions)

B. Bernoulli Confidence Interval

C. Binomial Confidence Interval

C1. Parameter/ probability / proportion

C2. Difference between two binomial parameters/probabilities/proportion

C3. Difference of two binomial proportions (for paired data)

C4. Ratio of binomial parameters/proportions

C5. Product of two binomials

D. Poisson Confidence Interval

D1. Parameter (mean or expectation) / rate / proportion

D2. Two Poisson rates

D3. Difference of two Poisson parameters

D4. Ratio of two Poisson means or parameters

E. Bayesian

F. Mid-p, Minimax, Fuzzy

G. Estimation

G1. Two rates

G2. Difference between two probabilities

G3. Binomial proportions

G4. Ratio of Poisson means

G5. Comparing Poisson processes (distributions)

G6. Several binomial proportions

H. Sample Size and Power

H1. For binomial parameter / proportion

H2. Difference between two probabilities

H3. For several binomial populations / proportions

H4. For two binomial experiments / parameters

H5. Comparing two independent binomial populations

H6. Comparing two Poisson rates

H7. Comparing two Poisson distributions

H8. Comparing several rates 
The codes or combinations of codes are inserted in square brackets at the end of each entry and adjacent to the right margin so that they can easily be scanned.

\section{References Cited:}

1. Agresti, A. (2002). Categorical Data Analysis, Second Edition, John Wiley, New York.

2. Agresti, A. (2007). An Introduction to Categorical Data Analysis, Second Edition, John Wiley, New York.

3. Casella, G. and Berger, R. L. (2002). Statistical Inference, Second Edition, Duxbury: Pacific Grove, CA.

4. Clopper, C. J. and Pearson, E. S. (1934). The use of confidence intervals or fiducial limits illustrated in the case of the binomial. Biometrika, 26, 404-413.

5. Davison, A. C. (2003). Statistical Models. Cambridge University Press, Cambridge.

6. Fisher, R. A. (1935). The fiducial argument in statistical inferences. Annals of Eugenics, 6, 391-398.

7. Fleiss, J. L., Levin, B., and Paik, M. C. (2003). Statistical Methods for Rates and Proportions, Third Edition, John Wiley, New York.

8. Hald, A. (2006). A History of Parametric Statistical Inference from Bernoulli to Fisher, 1713-1935. Springer-Verlag New York.

9. Hirji, K. F. (2006). Exact Analysis of Discrete Data. CRC Press/Chapman and Hall, New York.

10. Johnson, N. L., Kemp, A. W. and Kotz, S. (2005). Univariate Discrete Distributions, Third Edition, John Wiley, New York.

11. Krishnamoorthy, K. (2004). Handbook of Statistical Distribution with Applications. CRC Press/Chapman and Hall, New York.

12. Machin, D., Campbell, M. J., Tan, S. B. and Tan, S. H. (2009). Sample Size Tables for Clinical Studies, Third Edition, John Wiley, New York.

13. Neyman, J. (1937). Outline of a theory of statistical estimation on the classical theory of probability. Philosophical Transactions of the Royal Society of London, Series A, Mathematical and Physical Sciences, 236, 333-380.

14. Neyman, J. (1935). On the problem of confidence intervals. Annals of Mathematical Statistics, 6, 111-116.

15. Neyman, J. (1941). Fiducial argument and the theory of confidence intervals. Biometrika, 32, 128-150.

16. Simonoff, J. S. (2003). Analyzing Categorical Data. Springer-Verlag, New York.

17. Weerahandi, S. (2003). Exact Statistical Methods for Data Analysis. Springer-Verlag, New York.

\section{Acknowledgements}

Authors thank Professor Robert Newcombe for making a number of constructive comments and suggestions which have greatly improved the introduction, coverage, and organization of the bibliography. Authors are also thankful to 
Professor Paul Kabaila for providing us a collection of some of his own references which has greatly aided the task of compiling this bibliography.

\section{Bibliography}

Abonyti-Toth, Zs. and Reiczigel, J. (2006). "Simultaneous test and confidence set for two binomial proportions", AppEcoEnvRes, 4, 189-194.

[C2]

Afshartous, D. (2008). "Sample size determination for binomial proportion confidence intervals: An alternative perspective motivated by a legal case", AmerStat, 62, 27-31.

[H1]

Agresti, A. (1999). "On logit confidence intervals odds ratio with small samples", Biomtrcs, 55, 597-602.

$[\mathrm{A4}, \mathrm{E}]$

Agresti, A. (2003). "Dealing with discreteness: Making 'exact' confidence intervals for proportions, differences of proportions, and odds ratios more exact", StatMedRsr, 12, 3-21.

[A3.1,A4 ]

Agresti, A. and Caffo, B. (2000). "Simple and effective confidence intervals for proportions and differences of proportions result from adding two successes and two failures", AmerStat, 54, 280-288.

[A2.1,A3.1,C1]

Agresti, A. and Gottard, A. (2007). "Nonconservative exact small-sample inference for discrete data", CompStDAna, 51, 6447-6458.

Agresti, A. and Coull, B. A. (1998). "Approximate is better than 'exact' for binomial proportions", AmerStat, 52, 119-126 (Letter to the Editor, 54, 88).

[D1]

Agresti, A. and Min, Y. (2001). "On a small-sample confidence intervals for parameters in discrete distributions", Biomtrcs, 57, 963-971.

[A3.1,A4,C1]

Agresti, A. and Min, Y. (2002). "Unconditional small-sample confidence intervals for the odds ratio", Biostats, 3, 379-386.

[C1]

Agresti, A. and Min, Y. (2005 a). "Simple improved confidence intervals for comparing matched proportions", StatMed, 24, 729-740.

[A3.1,A4,C1]

Agresti, A. and Min, Y. (2005 b). "Frequentist performance of Bayesian confidence intervals for comparing proportions in $2 \times 2$ contingency tables", Biomtrcs, 61, 515523.

$[\mathrm{A} 3.1, \mathrm{~A} 4, \mathrm{C} 1]$

Agresti, A., Bini, M., Bertaccini, B. and Ryu, E. (2008). "Simultaneous confidence intervals for comparing binomial parameters", Biomtrcs, 64, 1270-1275. [A3.1,A4,C1]

Albert, J. H. (1983). "A pseudo-Bayes confidence region for $p$ Poisson means", JStatCompSim, 17, 11-29.

[A5,D1]

Altman, D. G. (1998). "Confidence intervals for the number needed to treat", BMJ, 317, 1309-1312.

Alvarez, O., Sotres, D. and Matuszewski, A. (1984). "A practical procedure to obtain confidence intervals for the Bernoulli parameter", CompStDAna, 2, 191-206.

Anbar, D. (1983). "On estimating the difference between two probabilities with special reference to clinical trials", Biomtrcs, 39, 257-262 (comments and reply (1984), 40, 1175-1176).

[A3.1,G2]

Anderson, T. W. and Burstein, H. (1967). "Approximating the upper binomial confidence limit", JASA, 62, 857-861.

[C1,D1]

Anderson, T. W. and Burstein, H. (1968). "Approximating the lower binomial confidence limit", JASA, 63, 1413-1415.

[C1,D1]

Angus, J. E. (1987). "Confidence coefficient of approximate two-sided confidence intervals for the binomial probability", NavResLog, 34, 845-851.

[C1]

Angus, J. E. and Schafer, R. E. (1984). "Improved confidence statements for the binomial parameter", AmerStat, 38, 189-191.

[C1] 
Arnett, L. M. (1976). "Confidence limits for parameters of Poisson and binomial distributions", Technical Report, Du Pont de Nemours (E.I.) and Co., Aiken, S.C. (USA). Savannah River Lab.

$[\mathrm{C} 1, \mathrm{D} 1]$

Austin, H. (1983). "An efficient procedure for computing exact confidence limits for a standardized mortality ratio", CompBioRsr, 16, 40-43.

[D1]

Bailey, B. J. R. (1987). "Confidence limits to the risk ratio", Biomtrcs, 43, 201-205. [A4]

Bain, L. J., Engelhardt, M. and Williams, D. H. (1990). "Confidence bounds for the binomial $N$ parameter: A classical approach", CommStatB, 19, 335-348.

[C1]

Bakirov, N. K. (1993). "An extremal property of the binomial distribution", MathMStat, 2 , 165-170.

$[\mathrm{A} 1, \mathbf{C} 1]$

Bapista, J. and Pike, M. C. (1977). "Exact two-sided confidence limits for the odds ratio in a $2 \times 2$ table", JRSS-C, 26, 214-220.

[A4]

Barker, L. (2002). "A comparison of nine confidence intervals for a Poisson parameter when the expected number of events is $\leq 5$ ", AmerStat, 56, 85-89 (comments, 56, 341).

[D1]

Barker, L. and Cadwell, B. L. (2008). "An analysis of eight 95 per cent confidence intervals for a ratio of Poisson parameters when events are rare", StatMed, 27, 40304037.

[D1]

Barker, L. and Cadwell, B. L. (2009). "Exact average coverage probabilities and confidence coefficients of confidence intervals for discrete distributions", StatComp, 19, 139-148.

[A1]

Barrowman, N. J. (2002). "Missing the point (estimate)? Confidence intervals for the number needed to treat", CMAI, 166, 1676-1677.

[A8]

Bartlett, M. S. (1953). "Approximate confidence intervals, II. More than one unknown parameter", Biomka, 40, 306-317.

[A1]

Bayer, J. (2005). "Binomial confidence intervals and diagnostics for binomial regression", M. Sc. Thesis, Department of Mathematics, Graduate School, Southern Illinois University Carbondale, USA.

[C1]

Beal, S. L. (1987). "Asymptotic confidence intervals for the difference between two binomial parameters for use with small samples", Biomtrcs, 43, 941-950.

[C2]

Beal, S. L. (1989). "Sample size determination for confidence intervals on the population mean and the difference between two population means", Biomtrcs, 45, 969-977. [ [H]

Bedrick, E. J. (1987). "A family of confidence intervals for the ratio of two binomial proportions", Biomtrcs, 43, 993-998.

$[\mathrm{A4}, \mathrm{C} 5]$

Bégaud, B., Martin, K., Abouelfath, A., Tubert-Bitter, P., Moore, N. and Moride, Y. (2005). "An easy to use method to approximate Poisson confidence limits", EuJEpi, 20, 213-216.

[C1]

Belyaev, Y. (1966). "On confidence intervals and sets for various statistical models", ProcFifthBerk, 1, 51-58.

[A1]

Bender, R. (2000). "Improving the calculation of confidence intervals for the number needed to treat", ProcMIE2000, pp. 29-32.

[A8]

Bender, R. (2001). "Calculating confidence intervals for the number needed to treat", ConCliTri, 22, 102-110.

[A8]

Benton, D. and Krishnamoorthy, K. (2002). "Performance of the parametric bootstrap method in small sample interval estimates", AdvAppStat, 2, 269-285.

[D1]

Berger, R. L. (1996). "More powerful tests from confidence interval p values", AmerStat, 50, 314-318.

Berger, R. L. and Coutant, B. W. (2001). "Letter to the Editor 'Small-sample interval estimation of Bernoulli and Poisson parameters'”, AmerStat, 55, 85.

Berger, R. L. and Doi, J. (2001). "Letter to the Editor", AmerStat, 55, 374.

[B,D1]

Berger, R. R. and Boos, D. D. (1994). "P values maximized over a confidence set for the nuisance parameter", JASA, 89, 1012-1016.

[A1] 
Berry, G. and Armitage, P. (1995). "Mid-p confidence intervals: A brief review", Ststcian, 44, 417-423.

Biggerstaff, B. J. (2008). "Confidence intervals for the difference of two proportions estimated from pooled samples", JABES, 13, 478-496.

$[A 3.1, C]$

Birnbaum, A. (1953). "Some procedures for comparing Poisson processes or populations", Biomka, 40, 447-449.

[G5]

Bityukov, S. I., Krasnikov, N. V. and Taperechkina, V. A. (2001). "Confidence Intervals for Poisson Distribution Parameter", available at http://eprintweb.org/S/authors/All/bi/Bityukov.

[D1]

Blaker, H. (2000). "Confidence curves and improved exact confidence intervals for discrete distributions", CanJStat, 28, 783-798.

$[\mathrm{A} 1, \mathrm{C} 1, \mathrm{D} 1]$

Blyth, C. R. (1986). "Approximate binomial confidence limits", JASA, 81, 843-855 (correction, 84, 636).

[C1]

Blyth, C. R. (1988). Comments on "Confidence intervals for $p$ based on a binomial $(n, p)$ random variable", CompStDAna, 7, 93-98.

[C1]

Blyth, C. R. and Hutchinson, D. W. (1960). "Tables of Neyman-shortness confidence interval's for the binomial parameter", Biomka, 47, 381-391.

[C1]

Blyth, C. R. and Still, H. A. (1983). "Binomial confidence intervals", JASA, 78, 108-116.

[C1]

Böhning, D. (1988). "Confidence intervals estimation of a rate and the choice of sample size", StatMed, 7, 865-875.

$[\mathrm{A} 2, \mathrm{H}]$

Böhning, D. (1994). "Better approximate confidence intervals for a binomial parameter", CanJStat, 22, 207-218.

Böhning, D. and Viwatwongkasem, C. (2005). "Revisiting proportion estimators", StatMedRsr, 14, 167-169.

$[\mathrm{A} 4, \mathrm{C} 1]$

Bolboaca, S. and Cadariu, A. C. (2003). "Binomial distribution sample confidence interval estimation 3: Post and pre test odds", LeoJSci, 2, 24-46.

[C1]

Bolboaca, S. and Jantschi, L. (2005). "Binomial distribution sample confidence interval estimation for positive and negative likelihood ratio medical key parameters", AMIAAnSymPro, pp. 66-70

[C1]

Bonett, D. G. and Price, R. M. (2006). "Confidence intervals for a ratio of binomial proportions based on paired data", StatMed, 25, 3039-3047 (comment, 26, 46844685).

[C5]

Boomsma, A. (2005). "Confidence intervals for a binomial proportion", Preprint available at www.ppsw.rug.nl/ boomsma/confbin.pdf

[C1]

Borges, L. C. and Furtado F. D. (2000). "A comparison among some approximate confidence intervals for binomial parameter through simulation", RevMatEst, 18, 9-26.

[C1]

Borkowf, C. B. (2006). "Constructing binomial confidence intervals with near nominal coverage by adding a single imaginary failure or success", StatMed, 25, 3679-3695.

[C1]

Bratcher, T. L. and Stamey, J. D. (2004). "A note on Bayesian interval estimation for comparing two Poisson rates", MathScntst, 29, 54-60.

[G5]

Brenner, D. J. and Quan, H. (1990). "Exact confidence limits for the binomial proportions - Pearson and Hartley revisited", Ststcian, 39, 391-397.

$[\mathrm{C} 1, \mathrm{E}]$

Brown, L. D. and Li, X. (2005). "Confidence intervals for two sample binomial distribution", JStatPlalnf, 130, 359-375.

[C]

Brown, L. D., Cai, T. T. and DasGupta, A. (2001). "Interval estimation for a binomial proportion (with discussion)", StatSc, 16, 101-133. 
Brown, L. D., Cai, T. T. and DasGupta, A. (2002). "Confidence intervals for a binomial proportion and asymptotic expansions", AnalStat, 30, 160-201.

[C1]

Brown, L. D., Cai, T. T. and DasGupta, A. (2003). "Interval estimation in exponential families", StatSin, 13, 19-49.

$[A 1, C 1, D 1, E]$

Buehler, R. J. (1957). "Confidence intervals for the product of two binomial parameters", JASA, 52, 482-493.

[A1]

Burstein, H. (1973). "Close approximation of binomial confidence limit at 10 confidence levels", CommSt, 2, 185-187.

[C1]

Burstein, H. (1973). "Close approximations of percentage points of the chi-square distribution and Poisson confidence limits", JASA, 68, 581-584 (correction, 69, 288).

[D1]

Burstein, H. (1975). "Finite population correction for binomial confidence limits", JASA, 70, 67-69.

[C1]

Burstein, H. (1989). "Transformed binomial confidence limits for listening tests", JAES, 37, 363-367.

[C1]

Burstein, H. and Burstein, N. (1990). "Binomial confidence limits adjusted for a confounding variable", CommStB, 19, 337-346.

[C1]

Byrne, J. and Kabaila, P. (2001). "Short exact confidence intervals for the Poisson mean", CommStA, 30, 257-261.

Byrne, J. and Kabaila, P. (2005). "Comparison of Poisson confidence intervals", CommStA, 34, 545-556.

[D1]

Cai, Y. (2005). "One-sided confidence intervals in discrete distributions", JStatPlalnf, 131, 63-88.

[A1]

Cai, Y. and Krishnamoorthy, K. (2005). "A simple improved inferential method for some discrete distributions", CompStDAna, 48, 605-621.

[A1]

Cai, Y. and Krishnamoorthy, K. (2006). "Exact size and power properties of five tests for multinomial proportions", CommStB, 35, 149-160.

[C1]

Campbell, M. (2005). "Confidence intervals and Newcombe's method - What are They?", Midwif, 21, 80-83.

[C1]

Casagrande, J. T., Pike, M. C. and Smith, P. G. (1978). "An improved approximate formula for calculating sample sizes for comparing two binomial distributions", Biomtrcs, 34, 483-486.

[H4]

Casella, G. (1986). "Refining binomial confidence intervals", CanJStat, 14, 113-129. [C1]

Casella, G. and Robert, C. (1989). "Refining Poisson confidence intervals", CanJStat, $17,45-57$.

[D1]

Cesana, B. M. (2004). "Sample size for testing and estimating the difference between two paired and unpaired proportions: a 'two-step' procedure combining power and the probability of obtaining a precise estimate", StatMed, 23, 2359-2373.

[H3]

Cepeda-Cuervoa, E., Aguilarb, W., Cervantesc, V., Corralesd, M., Díaze, I. and Rodríguezf, D. (2008). "Confidence Intervals and Credibility Intervals for a Proportion", RevColEst, 31, 211-228 (in Spanish).

[C1]

Cesana, B. M., Reina, G. and Marubini, E. (2001). "Sample size for testing a proportion in clinical trials: A 'two-step' procedure combining power and confidence interval expected width", AmerStat, 55, 288-292.

[H]

Chan, I. S. F. and Zhang, Z. (1997). "Confidence intervals for binomial data in small samples", ASAProBiop, pp. 212-217.

[C1]

Chan, I. S. F. and Zhang, Z. (1999). "Test-based exact confidence intervals for the differences of two binomial proportions", Biomtrcs, 55, 1202-1209.

[C2]

Chang, M. N. (2004). "Improved confidence intervals for a binomial parameter following a group sequential phase II clinical trial”, StatMed, 23, 2817-2826.

[C1] 
Chapman, D. G. (1952). "On tests and estimates for the ratio of Poisson means", AnallnsStMa, 4, 45-49.

[G4]

Chartier, F. (1978). "Validity of the Poisson approximation for the upper bound of a confidence interval relative to a proportion", RevStatApp, 26, 37-43 (in French). [A5]

Chartier, F. and Morice, E. (1977). "Validity of the Poisson approximation for the bounds of the confidence interval for a population", RevStatApp, 25, 27-32 (in French). [A5]

Chen, H. (1990). "The accuracy of approximate intervals for a binomial parameter", JASA, 85, 514-518.

[C1]

Chen, X. (2002). "A quasi-exact method for the confidence intervals of the difference of two independent binomial proportions in small sample cases", StatMed, 21, 943-956.

[C2]

Chen, X. (2007 a). "Exact computation of minimum sample size for estimation of binomial parameters," Preprint.

$[\mathrm{H}]$

Chen, X. (2007 b). "Exact computation of minimum sample size for estimation of Poisson parameters," Preprint.

Chen, X. (2007 c). "Exact computation of minimum sample size for estimating proportion of finite population," Preprint.

Chen, X. (2007 d). "Minimum coverage probabilities of confidence intervals", Preprint.

[C1, D1]

Chen, X., Zhou, K. and Aravena, J. L. (2004). "On the binomial confidence intervals and probabilistic robust control", Autca, 40, 1787-1789.

[C1]

Chen, X., Zhou, K. and Aravena, J. L. (2008). "Explicit formula for constructing binomial confidence interval with guaranteed coverage probability", CommStB, 37, 1173-1180.

[C1]

Chernick, M. R. and Liu, C. Y. (2002). "The saw-toothed behavior of power versus sample size and software solutions: Single binomial proportion using 'exact' methods", AmerStat, 56, 149-155.

[A5]

Cho, H. and Govindarajulu, Z. (2008). "'Sequential confidence limits for the ratio of two binomial proportions", IntJStatManSys, 3, 26-42.

Clunies-Ross, C. W. (1958). "Interval estimation for the parameter of binomial distribution", Biomka, 45, 275-279.

[C1]

Coe, P. R. and Tamhane, A. C. (1993). "Small sample confidence intervals for the difference, ratio and odds ratio of two success probabilities", CommStB, 22, 925-938.

$[A 1, A 2, A 3, A 4]$

Cohen, G. R. and Yang, S. Y. (1994). "Mid-p confidence intervals for the Poisson expectation", StatMed, 13, 2189-2203.

[F,D1]

Conlon, M. and Thomas, R. G. (1990). "A new confidence interval for the difference of two binomial proportions", CompStDAna, 9, 237-241.

[C4]

Conner, R. J. (1987). "Sample size for testing differences in proportions for the pairedsample design", Biomtrcs, 43, 207-211.

[C5]

Copas, J. B. (1992). "Exact confidence limits for binomial proportions - Brenner and Quan revisited", Ststcian, 41, 569-572.

[C1]

Correa, J. C. (2007). "Interval estimation for the Poisson distribution parameter with a single observation", RevColEst, 30, 69-75.

[D1]

Correa, J. C. and Sierra, E. (2001). "Confidence intervals for the parameter of the binomial distribution", RevColEst, 24, 59-72 [In Spanish].

[C1]

Correa, J. C. and Sierra, E. (2003). "Confidence intervals for the comparison of two proportions", RevColEst, 26, 61-75 [In Spanish].

[A2.2]

Couey, H. M. and Chew, V. (1986). "Confidence limits and sample size in quarantine research", JEcoEnt, 79, 887-890.

$[\mathrm{C} 1, \mathrm{D} 1, \mathrm{H}]$

Cousins, R. D. (1998). "Improved central confidence intervals for the ratio of Poisson means", NuclMPhyRsr, 417, 391-399. 
Cousins, R. D., Hymesa, K. E. and Tucke, J. (2010). "Frequentist evaluation of intervals estimated for a binomial parameter and for the ratio of Poisson means", NuclMPhyRsr, 612, 388-398.

$[\mathrm{C} 1, \mathrm{D} 4]$

Cressie, N. (1978). "Testing for the equality of two binomial proportions", AnallnsStMa, 30, 421-427.

Crow, E. L. (1956). "Confidence intervals for a proportion", Biomka, 43, 423-435. [C1]

Crow, E. L. (1975)." Confidence limits for small probabilities from Bernoulli trials", CommStat, 4, 397-414.

[B1]

Crow, E. L. and Gardner, R. S. (1959). "Confidence intervals for the expectation of a Poisson variable", Biomka, 46, 441-453.

[C1]

D'Agostino, R. B., Chase, W. and Belanger, A. (1988). "The appropriateness of some common procedures for testing the equality of two independent binomial population", AmerStat, 42, 198-202 (comments and reply, 43, 187-188).

[G1]

Daly, L. (1992). "Simple SAS macros for the calculation of exact binomial and Poisson confidence limits", CompBioMed, 22, 351-361.

$[\mathrm{C} 1, \mathrm{D} 1]$

Daly, L. E. (1998). "Confidence limits made easy: Interval estimation using a substitution method", AmerJEpid, 147, 783-790.

$[\mathrm{C} 1, \mathrm{D} 1]$

Dann, R.S. and, Koch, G. G. (2005). "Review and evaluation of methods for computing confidence intervals for the ratio of two proportions and considerations for noninferiority clinical trials", JBiopharmStat, 15, 85-107.

[C4]

Detre, K. and White, C. (1970). "The comparison for two Poisson-distributed observations", Biomtrcs, 26, 851-854.

[G5]

Dobson, A. J., Kuulasmaa, K., Eberle, E. and Scherer, J. (1991). "Confidence intervals for weighted sums of Poisson parameters", StatMed, 10, 457-462.

[D1]

Doi, J. and Berger, R. L. (2003). "Exact confidence interval for the difference of two binomial proportion", ASAProBiop, pp. 1256-1263.

[C2]

Dozzi, M. and Riedwyl, H. (1984). "Small sample properties of asymptotic tests for two binomial proportions", BiomtrcJ, 26, 505-516.

[G3]

Dudley, R. M. (2009). "Confidence intervals for the binomial parameter $p$ ", available at http://www-math.mit.edu/ rmd/443/PS2/binom-confintervals.pdf

[C1]

Duffy, D. E. and Santner, I. J. (1987). "Confidence intervals for a binomial parameter based on multistage tests", Biomtrcs, 43, 81-93.

Durgan, T., Bolboaca, S., Jantschi, L. and Achima, C. A. (2003). "Binomial distribution sample confidence intervals 1. Sampling and medical key parameters calculation", LeoEJPrac, 3, 45-74.

Eberhardt, K. R. and Flinger, M. A. (1977). "A comparison of two tests for equality of two proportions", AmerStat, 31, 151-155.

[G2]

Ederer, F. and Mantel, N. (1974). "Confidence limits on the ratio of two Poisson variables", AmerJEpid, 100, 165-167.

[D6]

Edwardes, M. D. (1998). "The evaluation of confidence sets with application to binomial intervals", StatSin, 8, 393-409.

[C1]

Fagan, T. (1996). "Quick BASIC program for exact and mid-P confidence intervals for binomial proportion", CompBioMed, 26, 263-267.

$[\mathbf{C} 1, \mathrm{~F}]$

Fagan, T. (1999). "Exact 95\% confidence intervals for differences in binomial proportions", CompBioMed, 29, 83-87.

[C1]

Falk, R. W. and Koch, G. G. (1998). "Testing a specified difference between proportions", Biomtrcs, 54, 1602-1614.

[A3]

Faulkenberry, G. D. (1970). "A note on tolerance limits for a binomial distribution", Technmcs, 12, 920-922.

[C1]

Fay, M. P. (2010). "Two-sided exact tests and matching confidence intervals for discrete data", RJour, 2, 53-58.

$[A, C 1]$ 
Fay, M. P. and Feuer, E. J. (1997). "Confidence intervals for directly standardized rates: a method based on the gamma distribution", StatMed, 16, 791-801.

[D1]

Feigin, P. D. and Lumelskii, P. (2000). "On confidence limits for the difference between two binomial parameters", CommStA, 29, 131-141.

[C3]

Fieller, E. C. (1954). "Some problems in interval estimation", JRSS-B, 16, 175-185. [A1]

Fowler, T. (2002). "Bootstrap confidence intervals for the binomial parameter: How good is their coverage when the sample size is a Poisson random variable?", M.S. Thesis, University of Colorado, Denver.

[C1, D1]

Fujino, Y. (1980). "Approximate binomial confidence limits", Biomka, 67, 677-681. [C1]

Fujino, Y. and Okumo, T. (1984). "The maximum average confidence limits for a binomial probability - one sided case", RptJUSE, 31, 1-7.

[C1]

Gail, M. and Gart, J. J. (1973). "The determination of sample sizes for use with the exact test in 2×2 comparative trials", Biomtrcs, 29, 441-448.

[H8]

Garcia-Perez, M. A. (2005). "On the confidence interval for the binomial parameter", QualQuan, 39, 467-481.

[C1]

Gart, J. J. (1971). "The comparison of proportions: A review of significance tests, confidence intervals and adjustments for stratification", RevIntStIns, 39, 148-169. [A1]

Gart, J. J. (1985). "Approximate tests and interval estimation of the common relative risk in the combination of $2 \times 2$ tables", Biomka, 72, 673-677.

[A6]

Gart, J. J. and Nam, J. (1988 a). "Approximate interval estimation of the ratio of binomial parameters: A review and corrections for skewness", Biomtrcs, 44, 323-338.

[C4]

Gart, J. J. and Nam, J. (1988 b). "Alternative confidence intervals estimation for the relative difference", Ststcian, 37, 427-431.

[A4]

Gart, J. J. and Nam, J. (1990). "Approximate interval estimation of the difference in binomial parameters: correction for skewness and extension to multiple tables", Biomtrcs, 46, 637-643.

[C2]

Garwood, F. (1936). "Fiducial limits for the Poisson distribution", Biomka, 28, 437-442.

[A6,D1]

Gelfand, A. E. and Thomas, D. L. (1974). "Confidence coefficients for approximate binomial interval estimates under hypergeometric models", AmerStat, 28, 52-54. [C1]

Geyer, C. J. and Meeden, G. D. (2005). "Fuzzy and randomized confidence intervals and p-values (with discussion)", StatSc, 20, 358-387.

[F]

Ghosh, B. K. (1979). "A comparison of some approximate confidence intervals for the binomial parameter", JASA, 74, 894-900.

[C1]

Ghosh, J. K. (1961). "On the relation among shortest confidence intervals of different types", CalStAssBull, 10, 147-152.

Goodall, G. (1995). “Don't get out of proportion”, TeachgSt, 17, 50-51.

[A1]

Gordon, I. (1994). "Sample size for two independent propotions: A review", AustJStat 36, 199-209.

[H3]

Graham, P. L., Mengersen, K. and Morton, A. P. (2003). "Confidence limits for the ratio of two rates based on likelihood scores: Non-iterative method", StatMed, 22, 2071-2083 (comment and reply, 23, 685-693).

[A2.1]

Greenland, S. (2001). "Simple and effective confidence intervals for proportions and differences of proportions result from adding two successes and two failures", AmerStat, 55, 172.

$[A 3, C]$

Gu, K., Ng, H. K. T., Tang, M. L. and Schucany, W. R. (2008). "Testing the Ratio of Two Poisson Rates", BiomtrcJ, 50, 283-298.

Guess, H. A. and Thomas, J. E. (1990). "A Rapidly Converging Algorithm for Exact Binomial Confidence Intervals about the Relative Risk in Follow-up Studies with Stratified Incidence-Density Data", Epid, 1, 75-77. 
Guess, H. A., Lydick, E. G., Small, R. D. and Miller, L. P. (1987). "Exact binomial confidence intervals for the relative risk in follow-up studies with sparsely stratified incidence density data", AmerJEpid, 125, 340-347.

[C1]

Hall, P. (1982). "Improving the normal approximation when constructing one-sided confidence intervals for binomial and Poisson parameters", Biomka, 69, 647-652.

$[\mathrm{C} 1, \mathrm{D} 1]$

Hannig, J. (2009). "On generalized fiducial inference”, StatSin, 19, 491-544.

[A6]

Hannig, J., lyer, H. K. and Patterson, P. (2006). "Fiducial generalized confidence interval", JASA, 101, 254-269.

[A6]

Harris, B. (1971). "Hypothesis testing and confidence intervals for products and quotients of Poisson parameters with applications to reliability", JASA, 66, 609-613. [D]

Harte, D. (2002). "Non asymptotic binomial confidence intervals", Preprint.

[C1]

Hauck, W. W. (1990). Confidence intervals for the seroprevalence determined from pooled sera", Technical Report, Department of Epidemiology and Biostatistics, University of California, San Franciso, USA.

Hauck, W. W. and Anderson, S. (1986). "A comparison of large-sample confidence interval methods for the difference of two binomial probabilities", AmerStat, 40, 318-322.

[C2]

Henderson, M. and Meyer, M. C. (2001). "Exploring the confidence interval for a binomial parameter in a first course in statistical computing", AmerStat, 55, 337-344.

[C1]

Hirji, K. F., Tan, S. J. and Elashoff, R. M. (1991). "A quasi-exact test for comparing two binomial proportions", StatMed, 10, 1137-1153.

Hutson, A. (2006). "Modifying the exact test for a binomial proportion and comparisons with other approaches", JAppStat, 33, 679-690.

[C1]

Huwang, L. (1995). "A note on the accuracy of an approximate interval for the binomial parameter", StatProbLett, 24, 177-180.

[C1]

Hwang, D-S. and Buehler, R. J. (1973). "Confidence intervals for some functions of several Bernoulli parameters with reliability applications", JASA, 68, 211-217. [B1,D1]

Iwasaki, M. and Hidaka, N. (2001). "Notes on the central and shortest confidence intervals for a binomial parameter", JapJBiom, 22, 1-13.

[C1]

Iwasaki, M. and Noriko, H. (2002). "Determination of sample sizes for use in constructing confidence intervals for a binomial parameter", JJapSocCStat, 15, 19-32.

$[\mathrm{C} 1, \mathrm{H1}]$

Iwasaki, M. and Hashigaki, S. (2004). "Confidence intervals for the difference between two independent binomial proportions", JFacEngSeiUni, 41, 9-20 (In Japanese). [C2] Jaech, J. L. (1970). "Comparing two methods of obtaining a confidence interval for the ratio of two Poisson parameters", Technmcs, 12, 383-387.

$[\mathrm{C} 1, \mathrm{D} 6]$

Jantschi, L. and Bolboaca, S. (2010). "Exact probabilities and confidence limits for binomial samples: Applied to the difference between two proportions", SciWorJ, 10, 865-878.

[C3]

Johnson, M. and Litaker, M. (2003). "A SAS program to calculate and plot widths of $(1-\alpha) \%$ confidence intervals for binomial proportions", Preprint.

[C2]

Jovanovic, B. D. (2005). "Binomial confidence intervals when no events are observed", EncyBiost, pp. 358-359.

[C1]

Jovanovic, B. D. and Levy, P. S. (1997). "A look at the rule of three", AmerStat, 51, 137-139.

[C1]

Jovanovic, B. D. and Viana, M. A. G. (1997). "Upper confidence bounds for binomial probability in safety evaluation", ASAProBiop, 140-144.

[C1]

Jovanovic, B. D. and Zalensky, R. (1997). "Upper bound on binomial probability when

the number of observed events is small or zero", AnlsEmMed, 30, 301-306.

[C1]

Julious, S. A. and Campbell, M. J. (1998). "Sample size calculations for paired or matched ordinal data", StatMed, 17, 1635-1642. 
Kabaila, P. (1998). "The choice of statistic on which to base tight upper confidence limits", AustNZJStat, 40, 189-196.

Kabaila, P. (2001). "Better Buehler confidence limits", StatProbLett, 52, 145-154. [A1]

Kabaila, P. (2003). "A large sample approximation to the profile plug-in upper confidence limit", ActAppMath, 78, 185-192.

Kabaila, P. (2005). "Computation of exact confidence intervals from discrete data using studentized test statistics", StatComp, 20, 401-414.

$[\mathrm{A} 1, \mathrm{C} 2]$

Kabaila, P. (2007). "Comparison of 'tail method' exact confidence limits for the difference of binomial probabilities", ActAppMath, 96, 283-291.

[C2]

Kabaila, P. (2008). "Statistical properties of exact confidence intervals from discrete data using studentized test statistics", StatProbLett, 78, 720-727.

$[\mathrm{A} 1, \mathrm{C} 1, \mathrm{D} 1]$

Kabaila, P. and Byrne, J. (2001 a). "Exact short Poisson confidence intervals", CanJStat, 29, 99-106.

[D1]

Kabaila, P. and Byrne, J. (2001 b). "Exact short confidence intervals from discrete data", AustNZJStats, 43, 303-309.

[A1,C1,D1]

Kabaila, P. and Lloyd, C. J. (1997). "Tight upper confidence limits from discrete data", AustNZJStat, 37, 193-204.

$[\mathrm{A1}, \mathrm{C1}, \mathrm{D} 1]$

Kabaila, P. and Lloyd, C. J. (2000 a). "Profile upper confidence limits from discrete data", AustNZJStat, 42, 67-79.

$[\mathrm{A1}, \mathrm{C1}, \mathrm{D} 1]$

Kabaila, P. and Lloyd, C. J. (2000 b). "A computable confidence upper limit from discrete data with good coverage properties", StatProbLett, 50, 115-120.

$[\mathrm{A} 1, \mathrm{D} 4]$

Kabaila, P. and Lloyd, C. J. (2000 c). "When do best confidence limits exist?", StatProbLett, 50, 115-120.

$[\mathrm{A} 1, \mathrm{C} 1, \mathrm{D} 1]$

Kabaila, P. and Lloyd, C. J. (2002). "The importance of the designated statistic on Buehler upper limits on a system failure probability", Technmcs, 44, 390-395.

[C5]

Kabaila, P. and Lloyd, C. J. (2003). "The efficiency of Buehler confidence limits", StatProbLett, 65, 21-28.

Kabaila, P. and Lloyd, C. J. (2004 a). "Buehler confidence limits and nesting", AustNZJStat, 46, 463-469.

[A1]

Kabaila, P. and Lloyd, C.J. (2004 b). "Buehler limits from approximate upper limits; a numerical investigation of the role of nominal coverage", JASS, 13, 217-230.

Kabaila, P. and Lloyd, C. J. (2005). "A simple measure of the efficiency of a Buehler confidence limit", CommStA, 34, 767-774.

[A1]

Kabaila, P. and Lloyd, C. J. (2006). "Improved Buehler limits based on refined designated statistics", JStatPlalnf, 136, 3145-3155.

[A1]

Kang, S.-H. and Chen, J. J. (2000). "An approximate unconditional test of non-inferiority between two proportions", StatMed, 19, 2089-2100.

[A3.3]

Katsis, A. (2001). "Calculating the optimal sample size for binomial populations", CommStA, 30, 665-678.

[H1]

Katz, D., Bapista, J., Azen, S. P. and Pike, M. C. (1978). "Obtaining confidence intervals for the risk ratio in cohort studies", Biomtrcs, 34, 469-474.

[A4]

Khurshid, A. and Sahai, H. (1996). "On confidence intervals for the binomial parameter in analyzing biomedical data", ProcSixthStatSemin, pp. 291-307.

[C1]

Kinsella, A. (1987). "The exact bootstrap approach to confidence intervals for the relative difference statistics", Ststcian, 36, 345-347.

[A4]

Koopman, P. A. R. (1984). "Confidence limits for the ratio of two binomial proportions", Biomtrcs, 40, 513-517.

[C1]

Kopit, J. S. and Berger, R. L. (1998). "A more powerful exact test for a practical difference between binomial proportions", ASAProBiop, pp. 251-256.

[C2]

Korn, E. L. (1986). "Sample size tables for bounding small proportions", Biomtrcs, 42, 213-216 (correction, 42, 691).

[C1,C2] 
Korn, E. L. and Graubard, B. I. (1998). "Confidence intervals for proportions with small expected number of positive counts estimated from survey data", TechEnq, 24, 209218.

$[\mathrm{C} 1, \mathrm{D} 1]$

Krishnamoorthy, K. and Lee, M. (2010). "Inference for functions of parameters in discrete distributions based on fiducial approach: Binomial and Poisson cases", JStatPlalnf, 140, 1182-1192.

[A6]

Krishnamoorthy, K. and Peng, J. (2007). "Some properties of the exact and score methods for binomial proportion and sample size calculation", CommStB, 36, 1171-1186.

$[\mathrm{C} 1, \mathrm{H} 1]$

Krisnamoorthy, K. and Thomson, J. (2004). "A more powerful test for comparing two Poisson means", JStatPlalnf, 119, 23-35.

Krishnamoorthy, K., Thomson, J. and Cai, Y. (2004). "An exact method of testing equality of several binomial proportions to a specified standard", CompStDAna, 45, 697-707.

[G6]

Kulkarni, P. M., Tripathi, R. C. and Michalek, J. E. (1998). "Maximum (Max) and Mid-P confidence intervals and $p$ values for the standardized mortality and incidence ratios", AmerJEpid, 147, 83-86.

[D1,F]

Kupper, L. L. and Hafner, K. B. (1989). "How appropriate are popular sample size formulas?", AmerStat, 43, 101-105.

Kwong, K-S. (1996). "A note on simultaneous confidence intervals for multinomial proportions", JStatCompSim, 55, 111-120.

[A5,D1]

Lachenbruch, P. A. (1969). "Tables of simultaneous confidence limits for the binomial and Poisson distributions", Biomka, 56, 452-452.

$[\mathrm{A} 5, \mathrm{C} 1, \mathrm{D} 1]$

Lai, D., Wang, S, and Hsi, B. P. (1999). "A comparative study of testing the equality of two binomial proportions: Normal approximations and beyond", FEJTStat, 3, 239-263.

Lamy, B., Delignette-Muller, M. L., Baty, F. and Carret, G. (2004). "Simple table for estimating confidence interval of discrepancy frequencies in microbiological safety evaluation", JMM, 56, 137-139.

[A1]

Lancaster, H. O. (1961). "Significance tests in discrete distributions", JASA, 56, 223-234.

Lawrence, B. and $\mathrm{Li}, \mathrm{X}$. (2005). "Confidence intervals for two sample binomial distribution", JStatPlanlnf, 130, 359-375.

[C3]

Lecoutre, B. and Faure, S. (2007). "A note on new confidence intervals for the difference between two proportions based on an Edgeworth expansion", JStatPlanInf, 137, 355356.

[A3.1]

Lee, M. (2010). "Fiducial inference for some discrete distributions", Ph. D. Thesis, University of Louisiana at Lafayette.

[A6]

Lee, S-C. (2006). "Interval estimation of binomial proportions based on weighted Polya posterior", CompStDAna, 51, 1012-1021.

[C1]

Lee, J. J., Serachitopol, D. M. and Brown, B. W. (1997). "Likelihood-weighted confidence intervals for the difference of two binomial proportions", BiomtrcJ, 39, 387-407. [C2]

Leemis, L. M. and Trivedi, K. S. (1996). "A comparison of approximate interval estimators for the Bernoulli parameter", AmerStat, 50, 63-68.

[B1,C1,D1]

Levin, B. and Chen, X. (1999). "Is the one-half continuity correction used once or twice to derive a well-known approximate sample size formula to compare two independent binomial distributions?", AmerStat, 53, 62-66.

[C2]

Lewis, J. R. (1996). "Binomial confidence intervals for small sample usability studies", AdvAppErg, pp. 732-737.

[C1]

$\mathrm{Li}$, Z. and Chuang-Stein, C. (2006). A note on comparing two binomial proportions in confirmatory noninferiority trials", DrInfJ, 40, 203-208.

[C4] 
Liddel, F. D. (1984). "Simple exact analysis of the standardized mortality ratio", JEpiCommHealth, 38, 85-88.

[D1]

Lin, C-Y. and Yang, M-C. (2006). "Improved exact confidence intervals for the odds ratio in two independent binomial samples", BiomtrcJ, 48, 1008-1019.

[C2,A4]

Lin, P. K. H., Richards, D. O., Long, D. R., Myers, M. D. and Taylor, J. A. (1983). "Tables for computing shortest confidence intervals involving the F-distribution", CommStB, 12, 711-725.

[D4]

Little, R. J. A. (1989). "Testing the equality of two independent binomial proportions", AmerStat, 43, 283-288.

[G3]

Liu, W. and Bailey, B. J. R. (2002). "Sample size determination for constructing a constant width confidence interval for a binomial success probability", StatProbLett, 56, 1-5.

$[\mathrm{C} 1, \mathrm{H}]$

Lloyd, C. J. (1990). "Confidence intervals from the difference between two correlated proportions", JASA, 85, 1154-1158.

[A3.2]

Lloyd, C. J. and Kabaila, P. (2003) "On the optimality and limitations of Buehler bounds", AustNZJStat, 45, 167-174.

[A1]

Lloyd, C. J. and Moldovan, M. V. (2007). "Exact one-sided confidence limits for the difference between two correlated proportions", StatMed, 26, 3369-3384.

[A3.2]

Louis, T. A. (1981). "Confidence interval for a binomial parameter after observing no success", AmerStat, 35, 154.

[C1]

Love, T. and Shah, S. (1998). "Comparing methods of computation for confidence intervals of binomial proportions", ASAProStatEdu, pp. 217-222.

[C1]

Lu, W-S. (2000). "Improved confidence intervals for a binomial parameter using the Bayesian method", CommStA, 29, 2835-2847.

$[\mathrm{C} 1, \mathrm{E}]$

Lumelskii, Y. (1969). "Confidence limits for linear function of unknown parameters", ThePrApp, 14, 364-367.

[A1]

Lunneborg, C. E. (2005). "Binomial confidence interval", EncyStatBehSci, pp.

[C1]

Lutsenko, M. M. and Maloshevskii, S. G. (2003). "Minimax confidence intervals for the binomial parameter", JStatPlalnf, 113, 67-77.

$[\mathbf{C} 1, \mathbf{F}]$

Madansky. A. (1962). More on length of confidence intervals", JASA, 57, 586-589. [A1]

Mandelkern, M. (2002). "Setting confidence intervals for bounded parameters", StatSc, 17, 149-172.

[D1]

Mandelkern, M. and Schultz, J. (2000). "A comment on the Roe-Woodroofe construction of Poisson confidence intervals", Preprint.

[D1]

Mantalos, P. and Zografos, K. (2008). "Interval estimation for a binomial proportion: a bootstrap approach", JStatCompSim, 78, 1249-1263.

[C2]

Mantel, N. (1988). "Exact limits on the ratio or difference of two independent binomial proportions", Biomtrcs, 44, 623.

[C2]

Marosi, J. and Tusnády, G. (1978). "On the constructions of confidence intervals for the expectation of a Poisson distribution on the basis of censored data", ConlnfTheo, Volume 7, pp. 347-352.

[A2.2]

Martin Andres, A. (1991). "A review of classic non-asymptotic methods for comparing two proportions by means of independent samples", CommStB, 20, 551-583. [A3.1]

May, W. L. and Johnson, W. D. (1997). "Confidence intervals for differences in correlated binary proportions", StatMed, 16, 2127-2136 (comment, 17, 2017-2021)

[A3.2]

Mehrotra, D. V., Chan, I. S. F. and Berger, R. L. (2003). "A cautionary note on exact unconditional inference for a difference between two independent binomial proportions", Biomtrcs, 59, 441-450.

[C2]

Mehta, C. R. and Walsh, S. J. (1992). "Comparison of exact mid-p and Mantel-Haenszel confidence intervals for the common odds ratio across several $2 \times 2$ contingency table", AmerStat, 46, 146-150.

$[\mathrm{A} 4, \mathrm{~F}]$ 
Mehta, C. R., Patel, N. R. and Gray, R. (1985). "Computing an exact confidence interval for the common odds ratio in several $2 \times 2$ contingency tables", JASA, 80, 969-973.

[A4]

Miao, W. and Gastwirth, J. L. (2004). "The effect of dependence on confidential intervals for a population proportion", AmerStat, 58, 124-130.

[A3]

Miettinen, O. S. and Nurminen, M. (1985). "Comparative analysis of two rates", StatMed, 4, 213-226.

[A2]

Molenaar, W. (1973). "Simple approximations to the Poisson, binomial, and hypergeometric distributions", Biomtrcs, 29, 403-407.

[A1]

Morice, E. (1977). "Use of the $F$ tables for computing a confidence interval for the parameter $p$ of a binomial," RevStatApp, 25, 33-38 (in French).

[C1]

Morisette, J. T. and Khorram, S. (1998). "Exact binomial confidence intervals for proportions", PEngRemSen, 64, 281-283.

[C1]

Mukhopadhyay, P. (2003). "Exact tests and exact confidence intervals for the ratio of two binomial proportions", Ph. D. Thesis, North Carolina State University.

[C2]

Mulder, P. G. (1983). "An exact method for calculating a confidence interval of a Poisson parameter (letter)", AmerJEpid, 117, 377.

[D1]

Nam, J. (1995). "Confidence limits for the ratio of two binomial proportions based on likelihood scores: Non-iterative method", BiomtrcJ, 37, 375-379.

[C4]

Nayatani, Y. and Kurahara, B. (1964). "A condition for using the approximation by the normal and the Poisson distribution to compute the confidence intervals for binomial parameter", RptJUSE, 11, 99-105.

[C1]

Nel, M., Schall, R., Nel, D. G. and Joubert, G. (2002). "A comparison of approximate confidence interval methods for the difference between two independent binomial proportions", SoAfrJStat, 36, 77-95.

[C2]

Nelson, L. S. (1981). "Confidence belts for small percentages", JQualTek, 13, 215-217.

[A1]

Nelson, L. S. (1989). "Upper confidence limits on average numbers of occurrences", JQualTek, 21, 71-72.

[D1]

Nelson. L. S. (1987). "Comparison of Poisson means: The general case", JQualTek, 19, 173-179.

[G5]

Nelson. L. S. (1991). "Power in computing Poisson means: II. Two sample test", JQualTek, 23, 162-166.

[G5]

Newcombe, R. G. (1994). "Confidence intervals for a binomial proportions", StatMed, 13, 1283-1285.

[C2]

Newcombe, R. G. (1998 a). "Two-sided confidence intervals estimation for the single proportion: Comparison of seven methods", StatMed, 17, 857-872.

$[\mathrm{A} 3, \mathrm{C} 1]$

Newcombe, R. G. (1998 b). "Interval estimation for the difference between two independent proportions: Comparison of eleven methods", StatMed, 17, 873-890 (comment, 18, 1293).

[A3,C1]

Newcombe, R. G. (1998 c). "Improved confidence intervals for the difference between binomial proportions based on paired data", StatMed, 17, 2635-2650 (comment and reply, 18, 3511-3513).

[C3]

Newcombe, R. G. (1999). "Confidence intervals for the number needed to treat: absolute risk reduction is less likely to be misunderstood", BMJ, 318, 1765.

[A8]

Newcombe, R. G. (2000 a). "Statistical applications in orthodontics, Part I; Confidence intervals: An introduction", JOrtho, 27, 270-272.

[A1]

Newcombe, R. G. (2000 b). "Statistical applications in orthodontics, Part II; Confidence intervals for proportions and their difference", JOrtho, 27, 339-340.

Newcombe, R. G. (2001 a). "Logit confidence intervals and the inverse sinh transformation”, AmerStat, 55, 200-202.

[C1] 
Newcombe, R. G. (2001 b). "Simultaneous comparison of sensitivity and specificity of two tests in the paired design: Straightforward graphical approach", StatMed, 20, 907-915.

[A5]

Newcombe, R. G. (2001 c). "Estimating the difference between differences: Measurement of additive scale interaction for proportions", StatMed, 20, 2885-2893.

[A3]

Newcombe, R. G. (2003). "Confidence intervals for the mean of a variable taking the values 0, 1 and 2", StatMed, 22, 2737-2750.

[C3]

Newcombe, R. G. (2008 a). "Confidence intervals for proportions: The Cinderella of statistical analyses", ClinOnc, 20, 92-93.

[A2]

Newcombe, R. G. (2008 b). "Nine out of ten cat owners ... Reporting proportions and related quantities", PubOpRes, pp. 123-134.

[A2]

Newcombe, R. G. and Altman, D. G. (2000 a). "Calculating confidence intervals for proportions and their differences", StatConf, pp. 28-33.

[A3]

Newcombe, R. G. and Altman, D. G. (2000 b). "Proportions and their differences", StatConf, pp. 46-48.

[A3]

Newcombe, R. G. and Soto, C. M. (2006). "Confidence intervals for estimations of proportions and their differences", IntRevPsi, 23, 141-154 (in Spanish).

[A3]

$\mathrm{Ng}, \mathrm{H}$. K. T. and Tang, M-L. (2005). "Testing the equality of two Poisson means using the rate ratio", StatMed, 24, 955-965.

[A4,D2]

$\mathrm{Ng}, \mathrm{H}$. K. T., Gu, K. and Tang, M-L. (2007). "A comparative study of tests for the difference of two Poisson means", CompStDAna, 51, 3085-3089.

[A4,D2]

Noether, G. E. (1957). "Two confidence intervals for the ratio of two probabilities and some measures of effectiveness", JASA, 52, 36-45.

[A2.2]

Nozawa, M., Okuno, T. and Hirai, K. (1992). "A study of the confidence intervals for a binomial proportion in special reference to true confidence and approximation", RptJUSE, 39, 1-20.

[C1]

Nurminen, M. (1986). "Confidence intervals for the ratio and difference of two binomial proportions (letter)", Biomtrcs, 42, 675-676.

[C2]

O'Fallon, J. R. (1993). "Using M \& Ms to teach binomial confidence interval estimation to medical researchers", ASAProStatEdu, pp. 258-265.

[C1]

Olivier, J. and May, W. L. (2006). "Weighted confidence interval construction for binomial parameters", StatMedRsr, 15, 37-46.

[C1]

Olivier, J and May W. L. (2007) "A discussion of binomial interval estimators on the boundary," JMissASci, 52, 178-183.

[C2]

Öz, M. (1997). "Confidence intervals for difference of two binomial proportions", HacBNatEng, Series B, 26, 245-265 (in Turkish).

[C2]

Pachares, J. (1960). "Tables of confidence limits for the binomial distribution", JASA, 55, 521-533.

[C1]

Pan, W. (2002). "Approximate confidence intervals for one proportion and differences of two proportions", CompStDAna, 40, 143-157.

[A3.1]

Parmet, Y. and Schechtman, E. (2007). "On a test of the difference between two binomial proportions", CommStA, 36, 887-896.

[G3]

Pastor, D. (2005). "On the coverage probability of the Clopper-Pearson confidence interval", TranElecComm, 50, 7-10.

[C1]

Patil, D. D. and Rajarshi, M. B. (2010). "Comparison of confidence intervals for the Poisson mean", ModAssStApp, 5, 79-91.

[G3]

Pena, E. A. and Lai, H. (2003). "Sample size determination for comparing two Poisson rates", Preprint.

[H7]

Peskun, P. H. (1990). "A note on a general method for obtaining confidence intervals from samples from discrete distributions", AmerStat, 44, 31-35.

[D2] 
Peskun, P. H. (1993). "A new confidence interval method based on the normal approximation for the difference of two binomial probabilities", JASA, 88, 656-661. [C2]

Pham-Gia, T. and Turkkan, N. (1993). "Bayesian analysis of the difference of two proportions", CommStA, 22, 1755-1771.

$[\mathrm{A3} .1, \mathrm{E}]$

Pham-Gia, T. and Turkkan, N. (2003). "Determination of exact sample sizes in the Bayesian estimation of the difference of two proportions", Ststcian, 52, 131-150. [E,H]

Piegorsch, W. W. (2004). "Sample sizes for improved binomial confidence intervals", CompStDAna, 46, 309-316.

$[\mathrm{C} 1, \mathrm{H} 4]$

Pires, A. M. and Amado, C. (2008). "Interval estimators for a binomial proportion: Comparison of twenty methods", REVSTAT, 6, 165-197.

[C1]

Pradhan, V. and Banerjee, T. (2008). "Confidence interval of the difference of two independent binomial proportions using weighted profile likelihood", CommStB, 37, 645-659.

Pratt, J. W. (1961). "The length of confidence intervals", JASA, 56, 549-567.

[C2]

Price, R. M. and Bonett, D. G. (2000). "Estimating the ratio of two Poisson rates", CompStDAna, 34, 345-356.

$[\mathrm{A} 2.1, \mathrm{D} 2]$

Price, R. M. and Bonett, D. G. (2004). "An improved confidence interval for a linear function of binomial proportions", CompStDAna, 45, 449-456.

[C1]

Price, R. M. and Bonett, D. G. (2008). "Confidence intervals for a ratio of two independent binomial proportions", StatMed, 27, 5497-5508.

[C4]

Pulskamp, R. J. (1990). "A note on the estimation of binomial probabilities", AmerStat, 44, 293-295.

[G3]

Puza, B. and O'Neill, T. (2006). “Generalised Clopper-Pearson confidence intervals for the binomial proportion", JStatComSim, 76, 489-508.

Rahme, E. and Joseph, L. (1998). "Exact sample size determination of binomial experiments", JStatPlalnf, 66, 83-93.

Ramsey, L. T. (2003). "(Traditional) exact confidence intervals for the binomial distribution", Preprint.

[C1]

Reed, J. F. (2007). "Non iterative confidence interval methods for the difference between two proportions", IntJEpid, 5, 2, available at www.ispub.com

[A3.1]

Reed, J. F. (2008). "Better binomial confidence intervals", JModAppStMeth, 6, 153-161.

[C1]

Reiczigel, J. (2003). "Confidence intervals for the binomial parameter: Some new considerations", StatMed, 22, 611-621.

[C1]

Reiczigel, J., Abonyi-Tóth, Z. and Singer, J. (2008). "An exact confidence set for two binomial proportions and exact unconditional confidence intervals for the difference and ratio of proportions", CompStDAna, 52, 5046-5053.

$[\mathrm{C2}, \mathrm{C} 8]$

Rice, W. R. (1988). "A new probability model for determining exact $p$-values for $2 \times 2$ contingency tables when comparing binomial proportions", Biomtrcs, 44, 1-22.

[H4]

Ricker, W. E. (1937). "The concept of confidence or fiducial limits applied to the Poisson frequency distribution", JASA, 32, 349-386.

$[A 6, D 1]$

Ross, T. D. (2003). "Accurate confidence intervals for binomial proportion and Poisson rate estimation", CompBioMed, 33, 509-531.

$[\mathrm{C} 1, \mathrm{D} 1]$

Roths, S. A. and Tebbs, J. M. (2006). "Revising Beal's confidence intervals for the difference of two binomial proportions", CommStatA, 35, 1593-1609.

[C2]

Rubin, D. B. and Schenker, N. (1987). "Logit-based interval estimation for binomial data using the Jeffreys prior", SocMeth, 17, pp. 131-144.

[C1]

Rutledge, J. and Warner, B. (1999). "Using the beta distribution on confidence intervals for proportions", Presented May 20th, 1999 Quality and Productivity Research Conference.

[A1]

Sahai, H. and Khurshid, A. (1993 a). "Confidence intervals for the mean of a Poisson distribution: A review", BiomtrcJ, 35, 857-867.

[D1] 
Sahai, H. and Khurshid, A. (1993 b). "Confidence intervals for the ratio of two Poisson means", MathScntst, 18, 43-50.

[D3]

Sahai, H. and Khurshid, A. (1996). "Confidence intervals for the probability of success in the binomial distribution: A review", Metron, LIV, 153-180.

Sahai, H. and Misra, S. C. (1992). "Comparing means of two Poisson distributions", MathScntst, 17, 60-67.

[G5]

Samuels, M. L. and Lu, T-F C. (1992). "Sample size requirements for the back-of-theenvelope binomial confidence interval", AmerStat, 46, 228-231.

$[\mathrm{C} 1, \mathrm{H} 2]$

Sandelius, M. (1952). "A confidence interval for the smallest proportion of a binomial population", JRSS-B, 14, 115-117.

[C1]

Santner, T. J. (1998). "Teaching large-sample binomial confidence intervals", TeachgSt, 20, 20-23.

[C1]

Santner, T. J. and Snell, M. K. (1980). "Small sample confidence intervals for $p_{1}-p_{2}$ and $p_{1} / p_{2}$ in $2 \times 2$ contingency tables", JASA, 75, 386-394.

[C2,C4]

Santner, T. J. and Yamagami, S. (1993). "Invariant small sample confidence intervals for the difference of two success probabilities", CommStB, 22, 33-59.

[C2]

Santner, T. J., Pradhan, V., Senchaudhri, P., Mehta, C. R. and Tamhane, A. (2007). "Small sample comparisons of confidence intervals for the difference of two independent binomial proportions", CompStDAna, 51, 5791-5799.

[C2]

Sato, T. (1990). "Confidence intervals for effect parameters common in cancer epidemiology", EnvHeaPer, 87, 95-101.

[A4]

Satterthwaite, F. E. (1957). "Binomial and Poisson confidence limits", IndQCon, 13, 5659.

[C1,D1]

Schader, M. and Schmid, F. (1990). "Charting small sample characteristics of asymptotic confidence intervals for the binomial parameter $p$ ", StatPap, 31, 251-264.

[C1]

Schoenberg, B. S. (1990). "Calculating confidence intervals for rates and ratios", NeurEpid, 2, 257-265.

[A2]

Schouten, H. J. A. (2002). "Simple and effective confidence intervals for the number needed to treat", ConCliTri, 100, 102. (Letter), 100-101.

[A8]

Schouten, H. J. A., Molenaar, J. W., Van Strik, R. and Boomsa, A. (1980). "Comparing two independent binomial proportions by a modified chi-square test", BiomtrcJ, 22, 241-248.

[C2]

Schwertman, N. C. (1996). "A connection between quadratic-type confidence limits and fiducial limits", AmerStat, 50, 242-243.

[A6]

Schwertman, N. C. and Martinez, R. A. (1994 a). "Approximate confidence intervals for the difference in two Poisson parameters", JStatCompSim, 50, 235-247.

[D2]

Schwertman, N. C. and Martinez, R. A. (1994 b). "Approximate Poisson confidence limits", CommStA, 23, 1507-1529.

[D1]

Sharma, R. (1975). "Bayes approach to interval estimation of a binomial parameter", AnlsMathStat, 27, 259-267.

$[\mathrm{C} 1, \mathrm{E}]$

Sheps, M. C. (1959). "An examination of some methods of comparing several rates or proportions", Biomtrcs, 15, 87-89.

[A3]

Shiue, W. and Bain, L. J. (1982). "Experiment size and power comparisons for twosample Poisson tests", JRSS-C, 31, 130-134.

[H6]

Sichel, H. S. (1973). "On a significance test for two Poisson variables", JRSS-C, 22, 50-58.

[H6]

Simon, G. (1996). "Z or t for binomial confidence intervals", TeachgSt, 18, 90-91. [C1]

Soms, A. P. (1989 a). "Exact confidence intervals, based on the $Z$ statistic, for the difference between two proportions", CommStB, 18, 1325-1341.

[A3] 
Soms, A. P. (1989 b). "Some recent results for exact confidence intervals for the difference between two proportions", CommStB, 18, 1343-1357.

[A3]

Song, W. T. and Chang, C-J. (2006). "Improved confidence intervals for the Bernoulli parameter", ASAProSurResMeth, pp. 3740-3747.

[B1,C1]

Song, W. T., Chang, C-J. and Liu, S-L. (2010). "Robust confidence intervals for the Bernoulli parameter", CommStA, 38, 3544-3560.

[B1,C1]

Springer, M. D. and Thompson, W. E. (1966). "Bayesian confidence limits for the product of $\mathrm{N}$ binomial parameters", Biomka, 53, 611-613.

$[\mathrm{C} 1, \mathrm{E}]$

Stamey, J. and Hamilton, C. (2006). "A note on confidence intervals for a linear function of Poisson rates", CommStB, 35, 849-856.

[D1]

Stamey, J. D., Boese, D. H. and Young, D. M. (2008). "Confidence intervals for parameters of two diagnostic tests in the absence of a gold standard", CompStDAna, 52, 1335-1346.

[D2]

Stamey, J., Young, D. M. and Bratcher, T. (2006). "Bayesian sample-size determination for one and two Poisson rate parameters with applications to quality control", JAppStat, 33, 583-594.

[H6]

Starr, T. B. (1983). "A method for calculating a confidence interval for the ratio of two Poisson parameters", AmerJEpid, 118, 785.

Stavereva, N., Stavrev, P. and Fallone, G. B. (2009). "Confidence limits of the probability of success in animal experiments and clinical studies: A Bayesian approach", PhyMed, 25, 43-46.

[C,E]

Sterne, T. E. (1954). "Some remarks on confidence or fiducial limits", Biomka, 41, 275-278.

[A1]

Stevens, W. L. (1950). "Fiducial limits of the parameter of a discontinuous distribution", Biomka, 37, 117-129.

[A6]

Stevens, W. L. (1957). "Shorter intervals for the parameter of the binomial and Poisson distributions", Biomka, 44, 436-440.

Storer, B. E. and Kim, C. (1990). "Exact properties of some exact test statistics comparing two binomial proportions", JASA, 85, 146-155.

[G3]

Suess, E. A., Sultana, D. and Gongwer, G. (2006). "How much confidence should you have in binomial confidence intervals?", Stats, 1, 3-7.

[C1]

Suissa, S. and Shuster, J. J. (1985). "Exact unconditional sampling sizes for the $2 \times 2$ binomial trial", JRSS-A, 148, 317-327.

[H1]

Sun, J. F., Santner, T. J. and Venard, C. (2002). "An empirical comparison of small sample confidence intervals for $p_{1}-p_{2}$ ", Technical Report, Ohio State University. [A3]

Sun, J., Ono, Y. and Takeuchi, Y. (1996). "A simple method for calculating the exact confidence interval of the standardized mortality ratio with an SAS function", JOccuHealth, 38, 196-197.

[D1]

Swift, M. B. (2009). "Comparison of confidence intervals for a Poisson mean - Further considerations", CommStA, 38, 748-759.

[D1]

Tanaka, M. (1962). "On a confidence interval of given length for the parameter of the binomial and the Poisson distributions", AnallnsStMa, 13, 201-215.

Tango, T. (1998). "Equivalence test and confidence interval for the difference in proportions for the paired-sample design", StatMed, 17, 891-908.

[C3]

Tebbs, J. M. and Bilder, C. (2003). "Confidence intervals for probability of disease transmission in multiple-vector-transfer designs", JABES, 9, 75-90.

[C1]

Tebbs, J. M. and Roths, S. A. (2008). "New large-sample confidence intervals for a linear combination of binomial proportions", JStatPlalnf, 138, 1884-1893.

[C1]

Thode, H. C. (1997). "Power and sample size requirements for tests of differences between two Poisson rates", Ststcian, 46, 227-230.

[H2] 
Thomas, D. G. (1971). "Algorithm AS 36: Exact confidence limits for the odd ratios in a $2 \times 2$ table", JRSS-C, 20, 105-110.

Thomas, D. G. and Gart, J. J. (1977). "A table of exact confidence limits for differences and ratios of two proportions and their odd ratios", JASA, 72, 73-76.

Tingley, M. and Li, C. (1993). "A note on obtaining confidence intervals for discrete parameters", AmerStat, 47, 20-23.

Troendle, J. F. and Frank, J. (2001). "Unbiased confidence interval for odds ratio of two independent samples with application to case-control data", Biomtrcs, 57, 484-489.

Tuyl, F. (2007). "Estimation of the binomial parameter: In defence of Bayes (1763)", Ph. $D$. Thesis, School of Mathematical and Physical Sciences, University of New Castle.

$[\mathrm{C} 1, \mathrm{E}]$

Ulicny, J. (2001). "A comparison of binomial proportion interval estimation methods", available at www.nesug.org/Proceedings/nesug01/ps/ps8021.pdf

[C1]

Ulm, K. A, (1990). "Simple method to calculate the confidence interval of a standardized mortality ratio (SMR)", AmerJEpid, 131, 373-375 (comments and reply, ibid, 133, 212214).

[D1]

Vollset, S. E. (1993). "Confidence intervals for a binomial proportion", StatMed, 12, 809824 (comment and reply, 13, 1693-1698).

[C1]

Vos, P. W. and Hudson, S. (2005). "Evaluation criteria for discrete confidence intervals: Beyond coverage and length", AmerStat, 59, 137-142.

[A1]

Vos, P. W. and Hudson, S. (2008). "Problems with binomial two-sided tests and the associated confidence intervals", AustNZJStat, 50, 81-89.

[C1]

Wald, A. (1943). "Tests for statistical hypotheses concerning several parameters when the number of observations is large", TrAmMaSo, 54, 426-482.

[C1]

Wallenstein, S. (1997). "A non-iterative accurate asymptotic confidence interval for the difference between two proportions", StatMed, 16, 1329-1336.

Waller, J. L., Addy, C. L., Jackson, K. L. and Garrison, C. Z. (1994). "Confidence intervals for weighted proportions", StatMed, 13, 1071-1082.

[C1]

Walters, D. E. (1985). "An examination of the conservative nature of classical confidence limits for a proportion", BiomtrcJ, 27, 851-861.

[C1]

Walton, G. S. (1970). "A note on nonrandomized Neyman-shortest unbiased confidence intervals for the binomial and Poisson parameters", Biomka, 57, 223-224. [C1,D1]

Wang, H. (2007). "Exact confidence coefficients of confidence intervals for a binomial proportion", StatSin, 17, 361-368.

[C1]

Wang, W. (2006). "Smallest confidence intervals for one binomial proportion", JStatPlanInf, 136, 4293-4306.

[C1]

Wang, W. (2010). "On construction of the smallest one-sided confidence interval for the difference of two proportions", AnalStat, 17, 361-368.

[A3.1]

Wardell, D. G. (1997). "Small-sample interval estimation of Bernoulli and Poisson parameters", AmerStat, 51, 321-325 (comment and reply, 55, 85).

[B1,D1]

Weissfeld, L. A., St. Laurent, R. T. and Moulton, L. H. (1991). "Confidence intervals for the comparison of two Poisson distributions", CommStB, 20, 3071-3081.

[D2]

Wendell, J. P. and Schmee, J. (2001). "Likelihood confidence intervals for proportion in finite population", AmerStat, 55, 55-61.

Weerahandi, S., (1993). "Generalized confidence intervals", JASA, 88, 899-905. [A7]

Wilson, E. B. (1927). "Probable inference, the law of succession, and statistical inference", JASA, 22, 209-212.

Witkovsky. V. (2002). "Computing the exact and mid-p interval estimates for the differences of two independent binomial proportions", available at http://www.mathpreprints.com. 
Woodward, J. A., Liu, W. C. and Bonett, D. G. (1997). "Shortest two-tailed confidence intervals", AppMathComp, 84, 65-76.

Yamazoe, S. (1992). "Confidence intervals for the binomial parameter", MemShiga, 41, 1-13 (in Japanese).

[C1]

Yamazoe, S. (1993). "Confidence belts for the binomial parameter", JouJStaSoc, 23, 161-169.

[C1]

Yeh, S-T. (1990). "Determining sample sizes needed to achieve the specified confidence levels -- Binomial distribution approach", ProcSASUsGr, Volume 15, pp. 1176-1179.

[H1]

Yoo, S. and David, H. T. (2004). "Revisiting Clopper-Pearson", Preprint, available at http://www.stat.iastate.edu/preprint/articles/2002-05.pdf

[C1]

Zhao, P-L., Troxell, J. K., Quan, H., Lee, M. and Bolognese, J. A. (2001). "Confidence interval for the difference in binomial proportions from stratified $2 \times 2$ samples", ASAProJSM.

[C2]

Zhao, S. (2005). "Statistical inference on binomial proportions", Ph.D. Thesis, Mathematical Sciences, Arts and Sciences, University of Cincinnati.

[C1]

Zheng, X. (1998). "Better saddlepoint confidence intervals via bootstrap calibration", ProcAmerMathSoc, Vol. 126, 3669-3679.

$[\mathrm{C} 1, \mathrm{D} 1]$

Zheng, X. and Loh, W-Y. (1995). "Bootstrapping binomial confidence intervals", JStatPlalnf, 43, 355-380.

[C1]

Zhou, X. H. and Qin, G. (2005 a). "A new confidence interval for the difference between two binomial proportions of paired data", JStatPlalnf, 128, 527-542.

[C3]

Zhou, X. H. and Qin, G. (2005 b). "Improved confidence intervals for the sensitivity at a fixed level of specificity of a continuous-scale diagnostic test", StatMed, 24, 465-477.

[C1]

Zhou, X. H., Qin, G. S. (2007). "A Supplement to 'A new confidence interval for the difference between two binomial proportions of paired data"', JStatPlanlnf, 137, 357358.

[C3]

Zhou, X. H., Li, C. M. and Yang, Z. (2008). "Improving interval estimation of binomial proportions", PhilTran, 366, 2405-2418.

[C1]

Zhou, X. H., Tsao, M. and Qin, G. (2004). "New intervals for the difference between two independent binomial proportions", JStatPlalnf, 123, 97-115.

[C2]

Zielinski, W. (2010). "The shortest Clopper-Pearson confidence interval for binomial probability", CommStB, 39, 188-193.

[C1]

Zou, G. and Donner, A. (2004). "A simple alternative confidence interval for the difference between two proportions", ConCliTri, 25, 3-12.

[A3]

Zou, Y. Z., Huang, W. and Zhang, X. (2009). "A note on confidence interval estimation for a linear function of binomial proportions", CompStDAna, 53, 1080-1085. 